\title{
Numerical Analysis of Pile-Soil Interaction under Axial and Lateral Loads
}

\author{
Yasser Khodair ${ }^{1}$, and Ahmed Abdel-Mohti ${ }^{2), *}$
}

(Received July 29, 2013, Accepted April 23, 2014)

\begin{abstract}
In this paper, the analysis of a numerical study of pile-soil interaction subjected to axial and lateral loads is presented. An analysis of the composite pile-soil system was performed using the finite difference (FD) software LPILE. Two three dimensional, finite element (FE) models of pile-soil interaction have been developed using Abaqus/Cae and SAP2000 to study the effect of lateral loading on pile embedded in clay. A lateral displacement of $2 \mathrm{~cm}$ was applied to the top of the pile, which is embedded into the concrete pile cap, while maintaining a zero slope in a guided fixation. A comparison between the bending moments and lateral displacements along the depth of the pile obtained from the FD solutions and FE was performed. A parametric study was conducted to study the effect of crucial design parameters such as the soil's modulus of elasticity, radius of the soil surrounding the pile in Abaqus/Cae, and the number of springs in SAP2000. A close correlation is found between the results obtained by the FE models and the FD solution. The results indicated that increasing the amount of clay surrounding the piles reduces the induced bending moments and lateral displacements in the piles and hence increases its capacity to resist lateral loading.
\end{abstract}

Keywords: pile-soil interaction, amount of soil, soil springs, LPILE, stiff soil.

\section{Introduction}

The soil-structure interaction in general has been a concern; therefore, more research is needed to further understand and better model this interaction (Abdel-Mohti and Pekcan 2013a, b), Khodair and Hassiotis (2013). The primary purpose of using piles is to transfer the loads from the superstructure and the abutment to a reliable soil, in cases where the soil near the ground surface can not support the applied loads. Piles can transfer both axial and lateral forces. As the pile is subjected to lateral loads, the soil mass surrounding the pile plays a key-role in providing lateral support for the pile. The nature of pile-soil interaction is three dimensional and to complicate the problem further, soil is a nonlinear and anisotropic medium. Therefore, finding a closed form solution to such problem is extremely difficult. Several methods have been used to predict the response of the composite pile-soil system. The persistent obstacle in such processes is to find a valid approximation for soil representation. The subgrade reaction approach provides the simplest solution for the pile-soil interaction problem. In this

\footnotetext{
${ }^{1)}$ Department of Civil Engineering and Construction, Bradley University, Peoria, IL 61625, USA.

${ }^{2)}$ Civil Engineering Department, Ohio Northern University, Ada, $\mathrm{OH} 45810$, USA.

*Corresponding Author; E-mail: a-abdel-mohti@onu.edu Copyright ( $\odot$ The Author(s) 2014. This article is published with open access at Springerlink.com
}

approach, the pile is treated as an elastic laterally loaded beam. The soil is idealized as a series of independent springs with constant stiffness, where the lateral stiffness at one point does not affect the lateral stiffness at other points along the depth of the pile. The spring stiffness, or modulus of subgrade reaction, is defined as the ratio of the soil reaction per unit length of the pile as described in Eq. (1):

$$
p=K_{h} y
$$

where $p$ is the soil resistance per unit length of the pile, $K_{h}$ is the modulus of subgrade reaction, and $y$ is the lateral deflection of the pile.

The behavior of the pile is assumed to follow the differential equation of a beam:

$$
E_{p} I_{p} \frac{d^{4} y}{d x^{4}}+K_{h} y=0
$$

where $x$ is length along pile, and $E_{p} I_{p}$ is the flexural stiffness of pile. The solution for the differential equation are readily available and can be found in Hetenyi (1946). The subgrade reaction has been widely accepted in the analysis of soilstructure interaction problems (Reese and Matlock 1956; Broms 1964). However, a drawback of the method is its inability to account for the continuity of soil. Additionally, the linear representation of the subgrade reaction for the soil elements along the depth of the pile fails to account for the non-linear nature of the soil. The p-y approach is another method for handling pile-soil interaction. The only difference between the p-y method and the subgrade reaction method is that the former is based on defining a nonlinear 
relationship between the soil reaction and the lateral deflection at each point along the depth of the pile. Therefore, a p-y relationship is defined at each distinctive point along the depth of the pile. The solution to Eq. (2) can be obtained using the finite difference method and computers. Appropriate boundary conditions must be imposed at the pile head to insure that the equations of equilibrium and compatibility are satisfied at the interface between the pile and the superstructure. The concept of a p-y curve was first introduced by McCelland and Focht (1958). The development of a set of $p-y$ curves can introduce a solution to the differential equation in Eq. (2), and provide a solution for the pile deflection, pile rotation, bending moment, shear, and soil reaction for any load capable of being sustained by the pile. Several methods to obtain p-y curves have been presented in the literature (Georgiadis and Butterfield 1982; O’Neill and Gazioglu 1984; Dunnavant and O’Neill 1989). These methods rely on the results of several empirical measurements. Some researchers such as Ruesta and Townsend (1997) and Gabr et al. (1994) have attempted to enhance p-y curve evaluation based on in situ tests such as cone penetration, pressuremeter and dilatometer. However, such attempts have focused on the soil part of soil pile interaction behaviors. Robertson et al. (1985) developed a method that used the results of a pushed in pressuremeter to evaluate $p$-y curves of a driven displacement pile. Attempts towards deriving $\mathrm{p}-\mathrm{y}$ curves using three dimensional finite element model has been provided by Brown Dan and Shie (1990, 1991). A simple elastic-plastic material model is used for the soil to model undrained static loading in clay soils. p-y curves are developed from the bending stresses in the pile, where nodal stresses along the pile are used to obtain bending. The finite element method (FEM) is considered the most powerful tool in modeling soil-structure interaction. The FEM has several advantages over the other methods, some of which are the: (1) versatility of the method allows for modeling different pile and soil geometries, (2) capability of using different boundary and combined loading conditions, (3) discretization of the model into small entities allows for finding solutions at each element and node in the mesh, (4) feasibility for modeling different types of soil models and various material behavior for piles, and (5) ability to account for the continuity of the soil behavior. Several researchers have used the FEM to model pile-soil interaction. Desai and Appel (1976) presented a finite element procedure that can allow for nonlinear behavior of soils, nonlinear interaction effects, and simultaneous application of axial and lateral loads. The pile was modeled as a one-dimensional beam element and the interaction between the pile and the soil was simulated by a series of independent springs. The variations of the generalized displacements and internal forces were described by means of energy functionals incorporating the adjoint structure concept. Thompson (1977) developed a two-dimensional finite element model to produce $\mathrm{p}-\mathrm{y}$ curves for laterally loaded piles. The soil was modeled as an elastic-hyperbolic material. Desai and Kuppusamy (1980) introduced a one dimensional finite element model, in which the soil was simulated as nonlinear springs and a beam column element for the pile. The Ramberg-Osgood model was used to define the soil behavior. Faruque and Desai (1982) implemented both numerical and geometric non-linearities in their threedimensional finite element model. The Drucker-Prager plasticity theory was adopted to model the non-linear behavior of the soil. The researchers declared that the effect of geometric non-linearity can be crucial in the analysis of pile-soil interaction. Kumar (1992) investigated the behavior of laterally loaded single piles and piles group using a threedimensional non-linear finite element modeling. Greimann et al. (1986) conducted a three dimensional finite element analysis to study pile stresses and pile-soil interaction in integral abutment bridges. The model accounted for both geometric and material nonlinearities. Nonlinear springs were used to represent the soil, and a modified RambergOsgood cyclic model was used to obtain the tangential stiffness of the nonlinear spring elements. Kooijman (1989) presented a quasi three-dimensional finite element model. The rationale behind his model was that for laterally loaded piles, the effect of the vertical displacements was assumed to be negligible. Therefore, it was plausible to divide the soil into a number of interacting horizontal layers. For these layers an elastoplastic finite element discretization was used. The contact algorithm in this model was based on defining an interface element, which characterized the tangential and normal behavior of pile and soil contact. This simulated slip, debonding, and rebonding of the pile and the soil. Bijnagte et al. (1991) developed a three-dimensional finite element analysis of the soil-structure interaction. The model utilized an elastic-perfectly plastic theory implementing the Tresca and the Mohr-Coulomb failure criteria. That paper introduced recommendations for the design of piles and design values for thermal expansion coefficients. Arsoy et al. (1999) developed a plane strain finite element model with symmetry around the centerline of the bridge. The abutment was modeled using linear stress-strain criteria. The approach fill and the foundation soil were modeled using hyperbolic material properties. The loads applied on the model represent the loads reflected from the superstructure and the abutment. Ellis and Springman (2001) developed a plane strain FE model for the analysis of piled bridge abutments. The study used an equivalent sheet pile wall having the same flexural stiffness per unit width as the piles and soil that it replaced. Faraji et al. (2001) used a three dimensional FE model to study the effect of thermal loading on pile-soil-interaction. The authors relied on the $\mathrm{p}-\mathrm{y}$ method to model the non-linear behavior of the soil. The soil pressure distribution on the abutment is typically nonlinear and varies with depth, amount, and mode of wall displacement. A small parametric study was conducted to study the effect of the level of soil compaction on the response of the composite pile-soil system. Rajashree and Sitharam (2001) developed a nonlinear finite element model of batter piles under lateral loading. In their model, the nonlinear soil behavior was modeled using a hyperbolic relation for static load condition and modified hyperbolic relation, including degradation and gap for cyclic load condition. 
The research described in this paper presents a numerical investigation to study the composite pile-soil system. The objectives of this research are to: (1) analyze pile-soil interaction using the finite difference software LPILE 2012 and the finite element software Abaqus/Cae and SAP2000, (2) compare the bending moments and lateral displacements induced along the depth of the pile using the finite difference method and the finite element models, and (3) conduct a parametric study to determine the effect of relevant design parameters which include the soil modulus of elasticity, increasing the amount of clay surrounding the piles, and varying the number of soil springs on the pile induced bending moment and lateral displacements along its depth.

\section{Bridge Description}

The bridge studied is a composite bridge. It consists of one span with span length of about $45.5 \mathrm{~m}$ and width of $32.2 \mathrm{~m}$. The reinforced concrete deck is approximately $25.4 \mathrm{~cm}$ thick and the spacing between stringers is $3.35 \mathrm{~m}$. The concrete abutment is supported using HP 14x89 A992 steel piles as shown in Fig. 1.

\section{Finite Difference Method Model}

A 2D finite difference (FD) model of the composite pilesoil system was developed using LPILE (2012). The soil profile consists of three layers; two layers of stiff clay without free water and one layer of weak rock (Fig. 2). However, an assumption of a single layer of stiff clay without water with a unit weight of $2001.2 \mathrm{~kg} / \mathrm{m}^{3}$, cohesion of $47.85 \mathrm{kPa}$, and a strain factor $\varepsilon_{50}=0.009$ is considered as a realistic representation of the soil. The pile is oriented such that bending is about the weak axis.

\section{Finite Element Models}

Two 3D finite element models were developed of the pilesoil interaction using the finite element software Abaqus/Cae and SAP2000.

In the Abaqus/Cae model, an elastic perfectly plastic model was adopted for modeling the piles with a modulus of

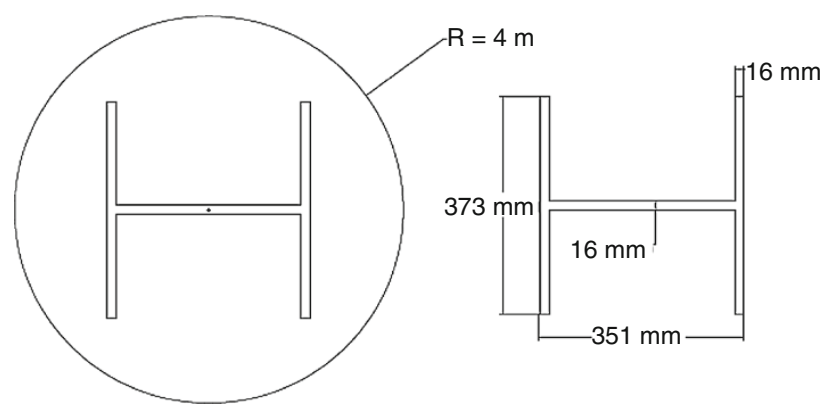

Fig. 1 a Cross section of HP $14 \times 89$ piles. b Cross-section of the sand sleeves surrounding the piles

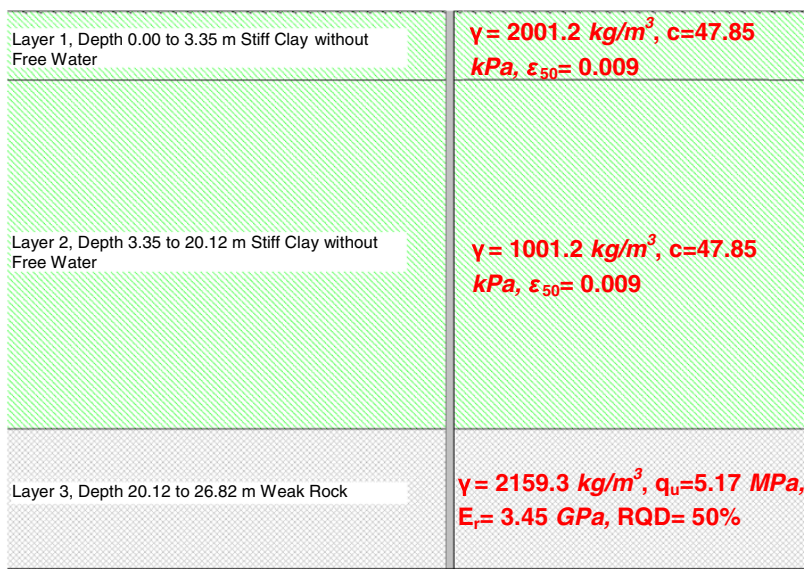

Fig. 2 Soil properties inputs in the FD LPILE model

elasticity of $200 \mathrm{GPa}$ and yield strength of $345 \mathrm{MPa}$. A single layer of stiff clay without free water was assumed. A strain hardening model using Mohr-Coulomb failure criterion was adopted for the soil with a variation in the modulus of elasticity of the clay in the range of (5-50 MPa) to represent the variation of the soil from soft to stiff clay and a possion's ratio of 0.4. An angle of internal friction of $20^{\circ}$ was used in the definition of the Mohr-Coulomb failure criterion. The interaction between the clay and the pile was modeled by defining tangential and normal contact behavior in the FE model. A master and slave surfaces were defined into the FE model as shown in Fig. 3. The master surface was represented by the exterior surface of the pile, and the slave surface by interior surface of the clay which was extruded according to the exact dimensions of the pile. The tangential contact between the two surfaces was defined using a friction coefficient of 0.36 . A relatively fine mesh was adopted for the pile and a coarser mesh was adopted for the clayey soil as shown in Fig. 4. In this model, the pile and clay were modeled using eight-nodded solid continuum elements (C3D8R) to account for the continuum nature of the soil in Abaqus/Cae. The bottom of the pile was fixed into the FE model to simulate the embedment of the pile into rock below a depth of $20.12 \mathrm{~m}$ and the exterior surface of the soil cylinder was fixed to model the confinement of the soil at its limits as shown in Fig. 5. The degrees of freedom of the elements at the top of the pile were restrained to a reference point defined at the centroid of the pile's cross section in what defines a rigid body motion to model the guided fixation occurring due to the embedment of the top of the pile into the concrete pile cap for a distance of $30.5 \mathrm{~cm}$.

In the SAP2000 model, an elastic three dimensional (3D) frame element was adopted for modeling the piles. The pile is made of A992 steel with a modulus of elasticity of $200 \mathrm{GPa}$ and yield strength of $345 \mathrm{MPa}$. The soil was modeled using nonlinear springs. The plastic (Wen) link element available in SAP2000 was used to model the hysteresis of soil. The springs were assigned in the longitudinal direction of the bridge. The nonlinear properties of the link elements were obtained using the generated p-y curves from the FD solution by LPILE. The number of soil springs were 


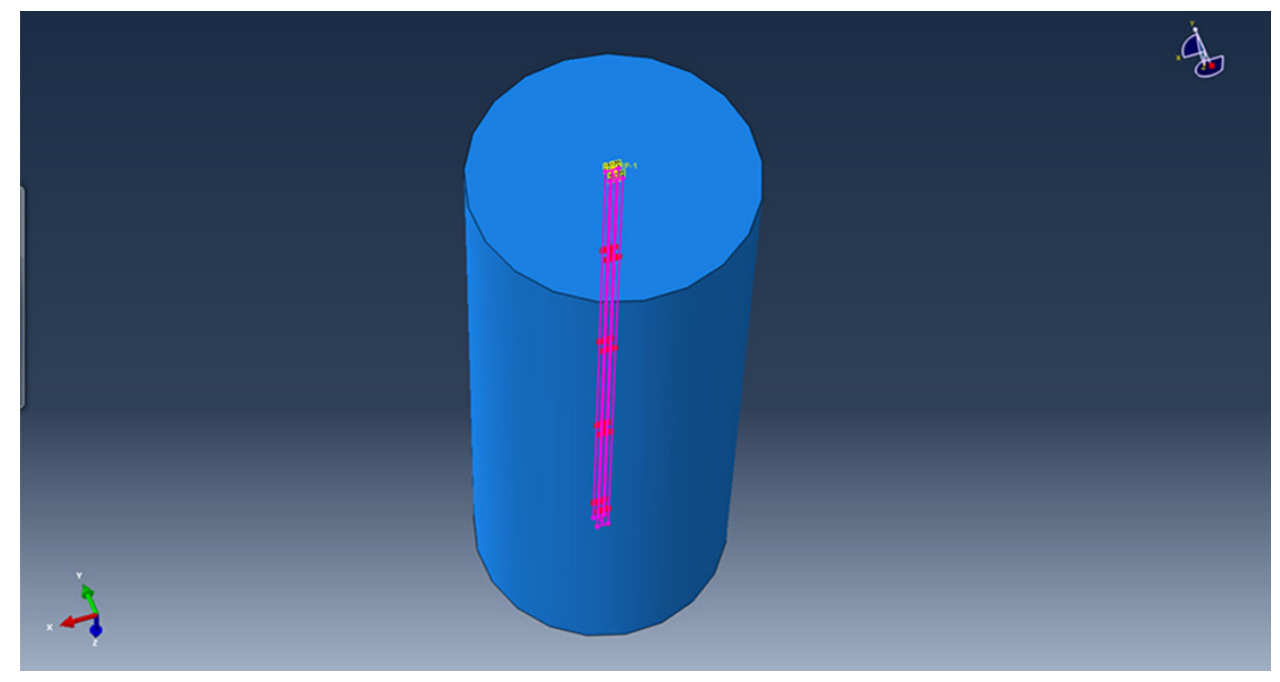

Fig. 3 Master and slave surfaces in Abaqus/Cae defining the contact behavior between pile and clay in the FE model

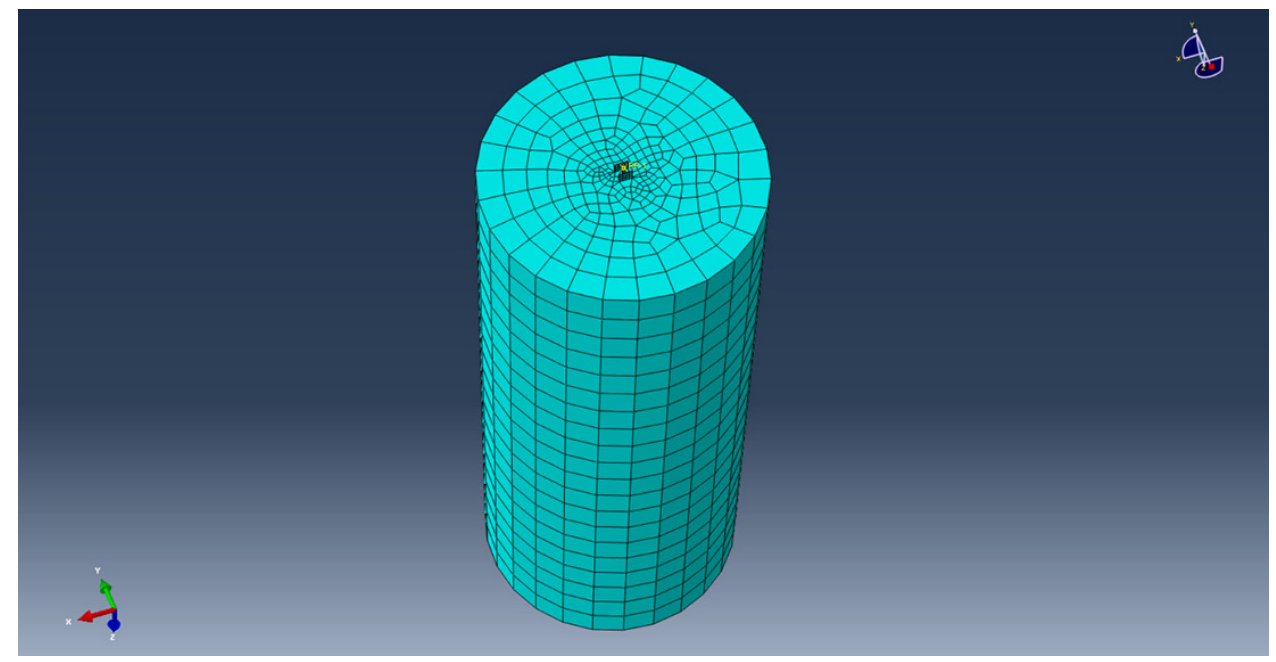

Fig. 4 FE mesh of pile

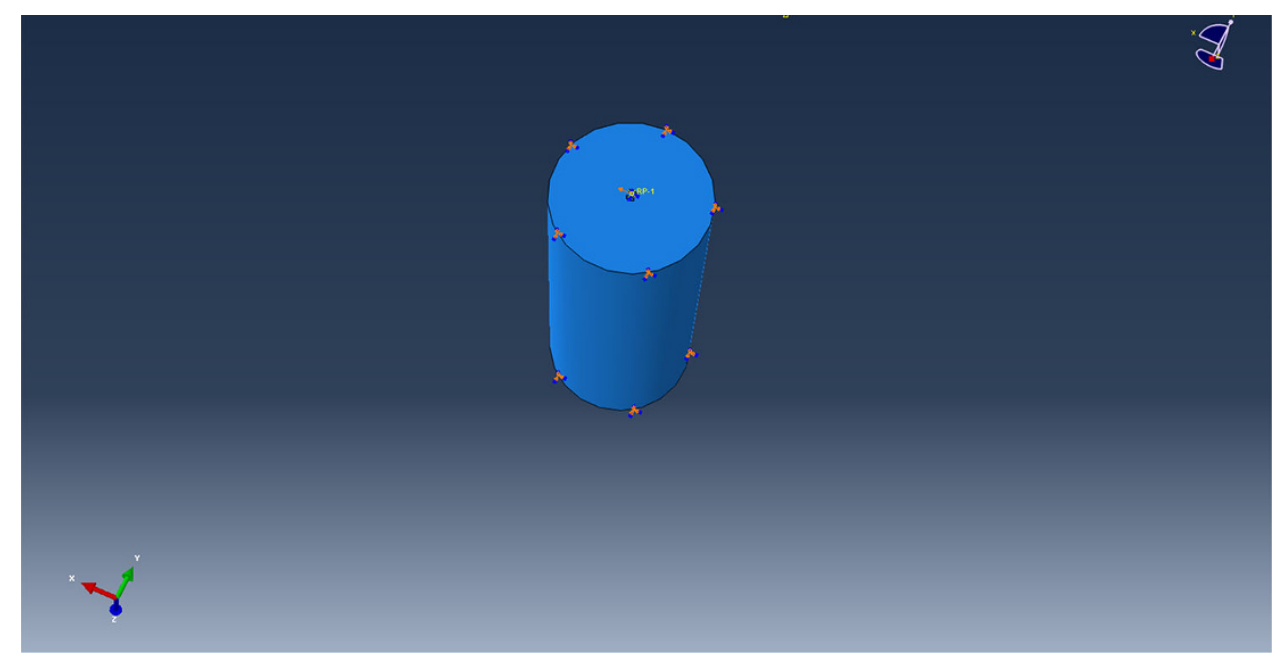

Fig. 5 Boundary Conditions used in Abaqus/Cae

varied to investigate the effect of changing the number of springs on the performance of the pile and to determine the proper number of springs that should be used. The three alternatives are using 7 springs, 9 springs, and 12 springs along the depth of the pile. In the 7 springs model, springs were assigned at $0,1.67,3.35,6.7,10.05,13.41$, and $16.76 \mathrm{~m}$ below the top of the pile. In the 9 springs model, springs were assigned at $0,0.91,1.83,2.74,3.35,6.7,10.05$,

242 | International Journal of Concrete Structures and Materials (Vol.8, No.3, September 2014) 
13.41 , and $16.76 \mathrm{~m}$ below the top of the pile. In the 12 springs model, springs were assigned at $0,0.91,1.83,2.74$, $3.35,3.96,4.57,5.18,6.7,10.05,13.41$, and $16.76 \mathrm{~m}$ below the top of the pile. The p-y curves were developed in LPILE at the defined depth locations and hence the soil stiffness at various depth locations was calculated and hysteretic behavior was obtained. Fixity was assigned at the bottom of the pile to simulate the embedment of the pile into rock below a depth of $20.12 \mathrm{~m}$ as shown in Fig. 6. The degrees of freedom of the elements at the top of the pile were restrained in a way to define a rigid body motion to model the guided fixation due to the embedment of the top of the pile into the pile cap for a distance of $30.5 \mathrm{~cm}$.

\section{Loading}

A displacement of $2 \mathrm{~cm}$ was applied to the reference point of the rigid body defined at the top of the pile to model the lateral displacement caused by thermal expansions and contractions at the top of the pile, while imposing a zero slope (guided-fixation). The effect of the axial load (298 kN) was applied as a vertical load at the reference point defining the rigid body motion at the top of the pile to study the effect

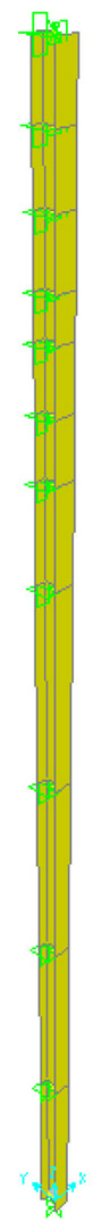

(a)

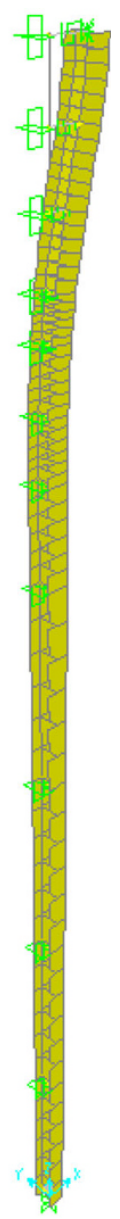

(b)
Fig. 6 Pile model overview using SAP2000. a Underfomed shape of pile and $\mathbf{b}$ deformed shape of pile due to a lateral displacement of $2 \mathrm{~cm}$ of including the axial load in addition to the lateral displacement on the induced bending moments and lateral displacements along the depth of the pile. Figures 6, 7, and 8 show the lateral displacement $\left(\mathrm{U}_{1}\right)$ and the lateral bending stress (S33) along the depth of the pile.

\section{Comparison Between the FE Models and LPILE}

The results obtained from the FE models (Abaqus/Cae and SAP2000) were compared to those produced by the FD model (LPILE, 2012). The bending moment and lateral displacement induced along the depth of the pile due to a lateral displacement of $2 \mathrm{~cm}$ applied at the top of the pile were compared using the three models for verification purposes. Figure 9 shows the close correlation between the results obtained by Abaqus/Cae and LPILE solutions. However, it shows that the inflection point for the pile's bending moment in Abaqus is slightly higher than that for LPILE. The reason for the discrepancy between the bending moments produced by Abaqus/Cae and LPILE is the variation in the soil definition in both approaches. Abaqus/Cae accounts for the continuum nature of the soil, while LPILE analysis is based on the discrete definition for the soil, where the stiffness of the soil at one point does not affect the other. This justifies the greater resistance of the soil in the FE model, which results in reversing the slope of the curve for the bending moment and hence the occurrence of the inflection point at a smaller depth below the top of the pile.

\section{Parametric Study}

A parametric study was conducted to analyze the effect of crucial design parameters such as the variation in the magnitudes of modulus of elasticity, the amount of soil surrounding the pile, and the number of soil springs on the bending moment and lateral displacements induced along the depth of the pile.

\subsection{Effect of Variation in Modulus of Elasticity}

The modulus of elasticity of the clay was varied from 5 to $50 \mathrm{MPa}$ to study the effect of the stiffness of the soil (soft to hard clay) under a lateral deformation of $2 \mathrm{~cm}$. Figure 10 shows that as the magnitude of the modulus of elasticity increases, the curves for the bending moments calculated by Abaqus/Cae and those produced by LPILE approach each other until the variation is minimal between the curves produced by both approaches when the soil's modulus of elasticity is in the range of $20-25 \mathrm{MPa}$. This modulus of elasticity corresponds to a medium to stiff soil which approximately matches the definition of the soil in LPILE. The reason for the discrepancy in the soil definition in both models can be attributed to the continuum nature of the soil in Abaqus/Cae. This continuity in the soil definition results in a smaller volume of soil needed to reverse the slope of the 


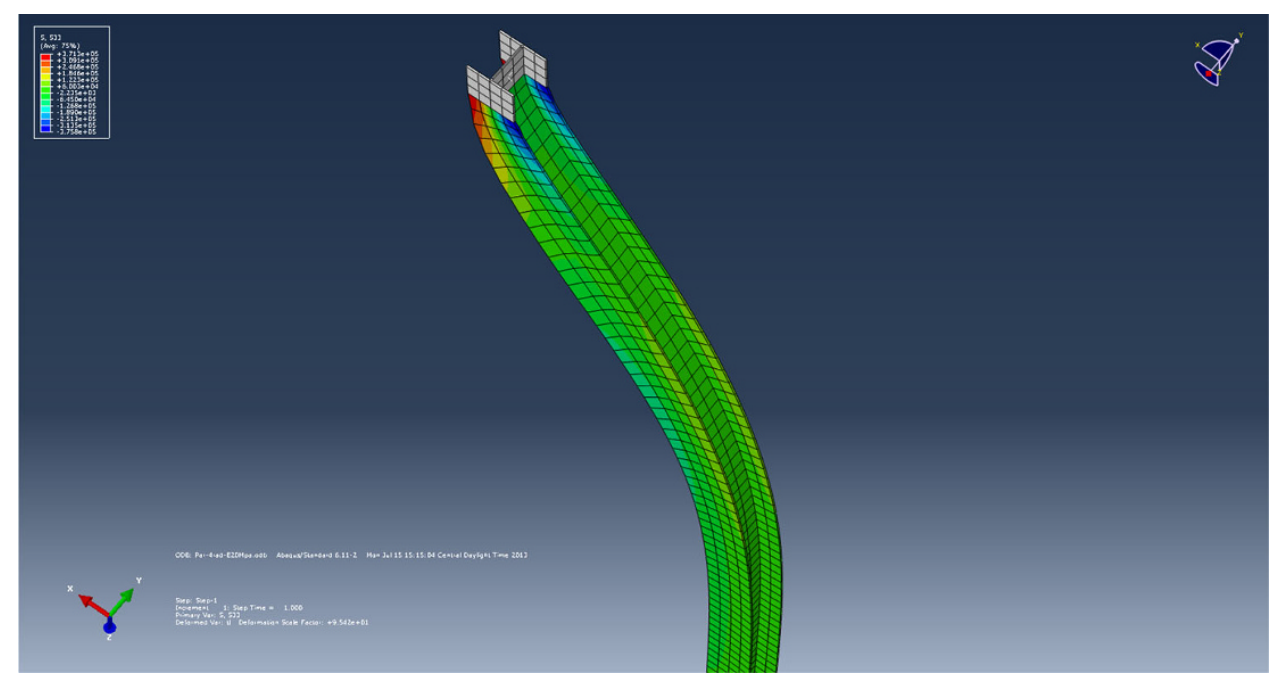

Fig. 7 A contour plot of pile bending stress, $S 33$ due to a lateral displacement of $2 \mathrm{~cm}$ at the top of the pile

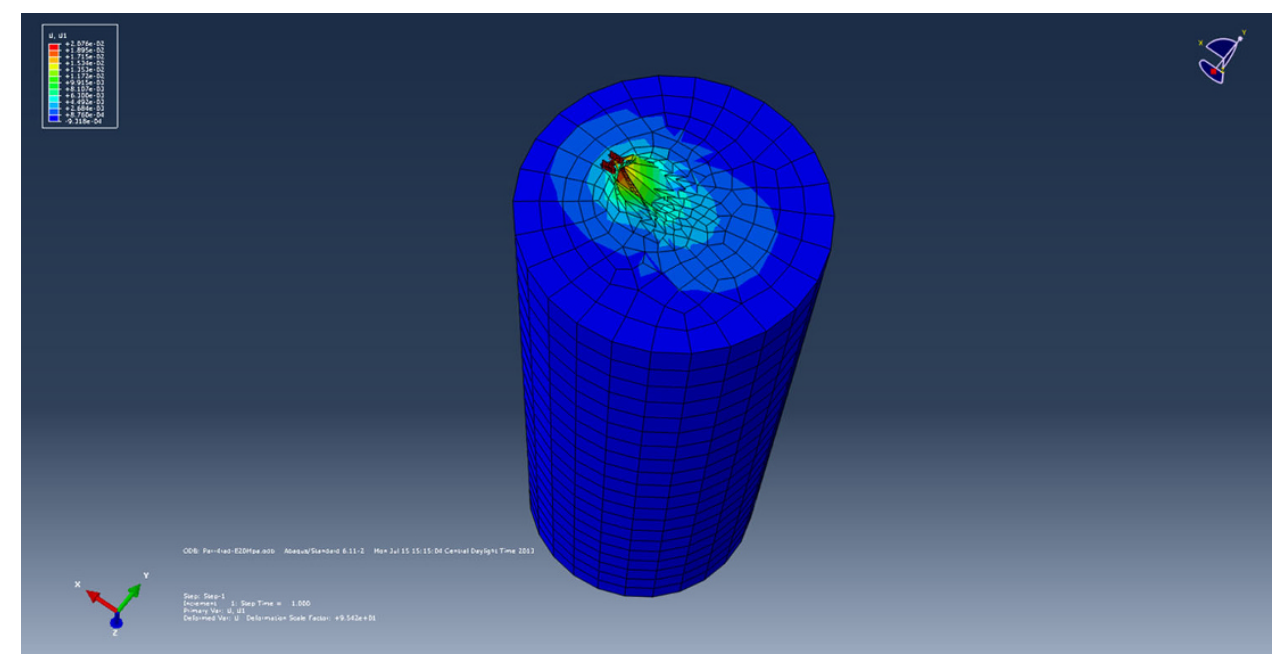

Fig. 8 A contour plot of pile lateral displacement, $U_{1}$ due to an imposed lateral displacement of $2 \mathrm{~cm}$ at the top of the pile

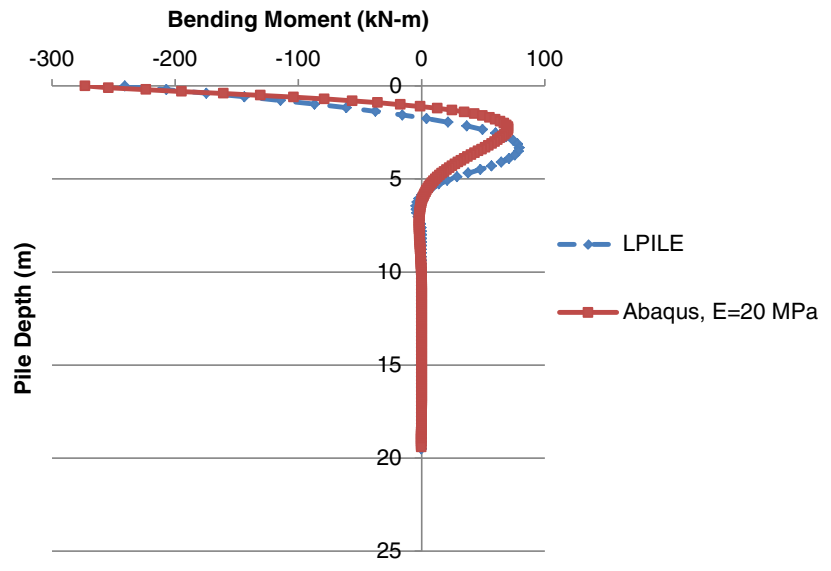

Fig. 9 Bending moment versus depth for pile-clay interaction due to a lateral displacement of $2 \mathrm{~cm}$

pile, and hence this occurs at a slightly smaller depth below the top of the pile than LPILE which is based on a discretization in the soil definition. At smaller or greater magnitudes of the modulus of elasticity, the discrepancy between the magnitudes of the bending moments and lateral

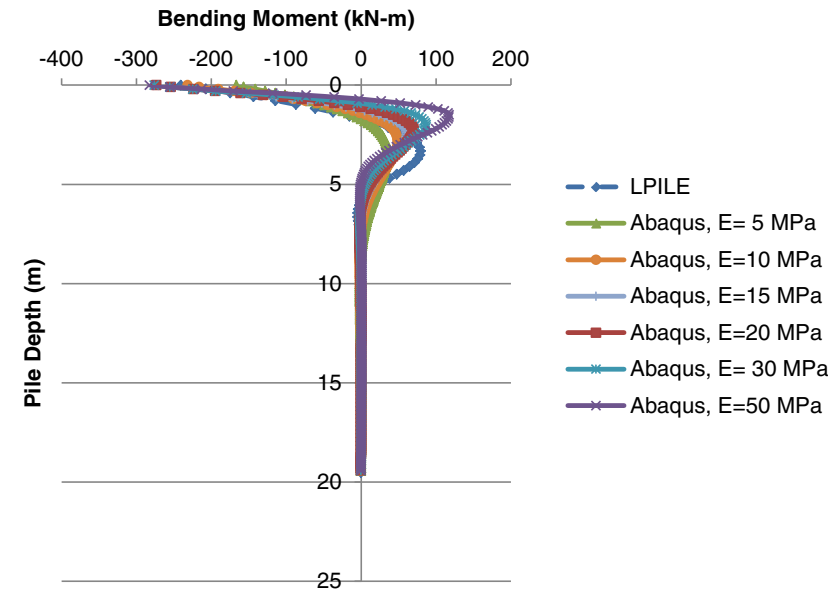

Fig. 10 Comparison between the bending moment versus pile depth obtained from the FE model Abaqus/Cae and FD solutions by LPILE at different clay moduli of elasticity due to a lateral displacement of $2 \mathrm{~cm}$

displacements increase due to adopting different clay stiffness in Abaqus/Cae and LPILE which is always based on a stiff clay definition for the soil. 


\subsection{Effect of Variation in Amount of Soil Surrounding the Pile}

The amount of clay medium surrounding the pile was studied by changing the radius of the soil cylinder surrounding the pile from 0.5 to $4 \mathrm{~m}$, while applying the same lateral displacement $(2 \mathrm{~cm})$ repeatedly. Figure 11 shows that as the radius of the soil surrounding the pile increases, the magnitudes of the positive bending moments decreases along the depth of the pile and the bending moments obtained from Abaqus/Cae approach those produced by LPILE for the same load. The same trend occurs for the lateral displacement along the depth of the pile (Fig. 12), as the radius of the clayey cylinder surrounding the pile increases, the lateral displacements decreases in magnitudes gradually until the values of the lateral displacements obtained from Abaqus/Cae become almost identical to those produced by LPILE at a radius of $4 \mathrm{~m}$ as shown in Fig. 12 .

\subsection{Effect of Variation in Number of Soil Springs}

In SAP2000, the soil was modeled using nonlinear springs that were assigned at different depths from the top of the pile. This approach is similar to that in LPILE since it is based on discrete definition of the soil, thus the soil is not modeled as a continuum media. The number of soil springs were varied to investigate the effect of changing the number of springs on the performance of the pile and to determine the proper number of springs that shall be used in order to model pile-soil interaction, adequately. Three alternatives were used including using 7 springs, 9 springs, and 12 springs. In the 7 springs model, springs were assigned at 0 , $1.67,3.35,6.7,10.05,13.41$, and $16.76 \mathrm{~m}$ below the top of the pile. In the 9 springs model, springs were assigned at 0 , $0.91,1.83,2.74,3.35,6.7,10.05,13.41$, and $16.76 \mathrm{~m}$ below the top of the pile. In the 12 springs model, springs were assigned at $0,0.91,1.83,2.74,3.35,3.96,4.57,5.18,6.7$, $10.05,13.41$, and $16.76 \mathrm{~m}$ below the top of the pile. The soil hysteretic properties such as yield strength and stiffness were calculated based on the p-y curves generated in LPILE at the defined depth locations (Fig. 13). Figure 14 shows that as

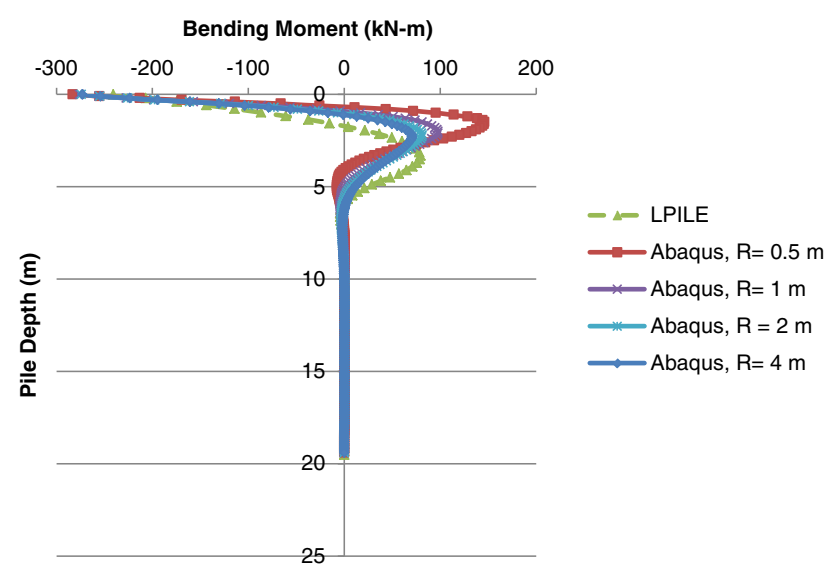

Fig. 11 Comparison between the bending moment versus pile depth obtained from the FE model Abaqus/Cae and FD solutions by LPILE at different soil cylinder radii due to a lateral displacement of $2 \mathrm{~cm}$

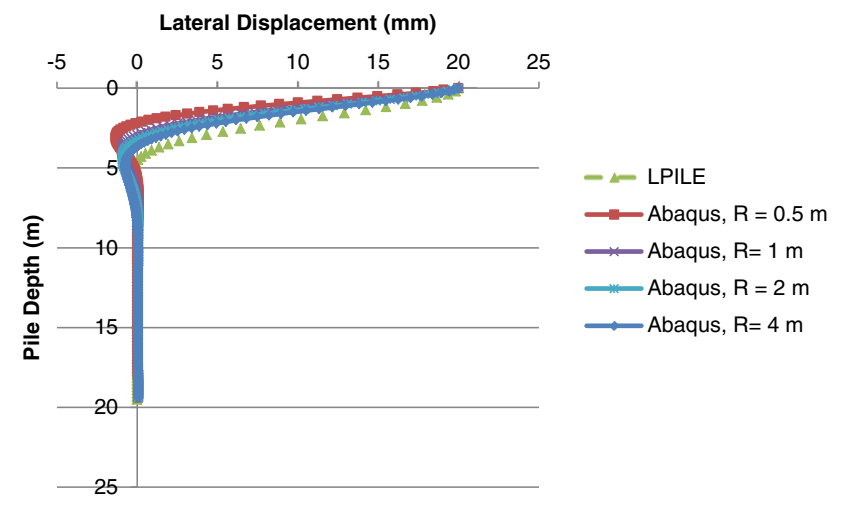

Fig. 12 Comparison between the lateral displacement versus pile depth obtained from the FE model Abaqus/Cae and FD solutions by LPILE at different soil cylinder radii due to a lateral displacement of $2 \mathrm{~cm}$

the number of springs increase, the magnitude of bending moment produced in the pile obtained from SAP2000 approach that obtained from LPILE. It can be observed that using 12 springs led to a close agreement between results of SAP2000 and LPILE owing to the similar approach used to define the soil in both of the software. Additionally, the magnitude of moment decreases moving downward from the top of the pile. Similar trend can be observed for the lateral displacement of the pile (Fig. 15). In the case of using 12 springs, the agreement between results of lateral displacements using SAP2000 and LPILE was fairly close, but not to the level observed in the case of bending moment. A better correlation can be obtained by using a more refined model through increasing the number of nonlinear soil springs, however, the agreement associated with using 12 springs was considered reasonable and acceptable.

\subsection{Effect of Applying Axial Load}

A comparison was conducted between LPILE, Abaqus/ Cae, and SAP2000 to study the effect of applying an axial load of $298 \mathrm{kN}$ to the pile on the produced bending moment and lateral displacement along the depth of the pile due to the applied displacement of $2 \mathrm{~cm}$ at the top of the pile. It seems that the applied axial load did not significantly affect neither the induced bending moment nor lateral displacement in the pile (Figs. 16 and 17). In Abaqus/Cae, applying the axial load did not show any significant effect on the induced bending moment and the results obtained from Abaqus/Cae closely matched those from LPILE (Fig. 16). In SAP2000, applying the axial load did not show an obvious effect on the induced bending moment and also results from SAP2000 closely matched those from LPILE (Fig. 17).

Kim and Jeong (2011) presented a study to investigate pile-soil interaction. They developed a series of 3D FE analyses. The analytical results and modeling methods that were used in this research were verified using results of field tests of large diameter laterally loaded piles in clay. The modulus of elasticity of soil ranges from 3 to $15 \mathrm{MPa}$. This range was covered in this research since the modulus of elasticity values used in this research ranges from 5 to $50 \mathrm{MPa}$. Lateral displacement and bending moment 


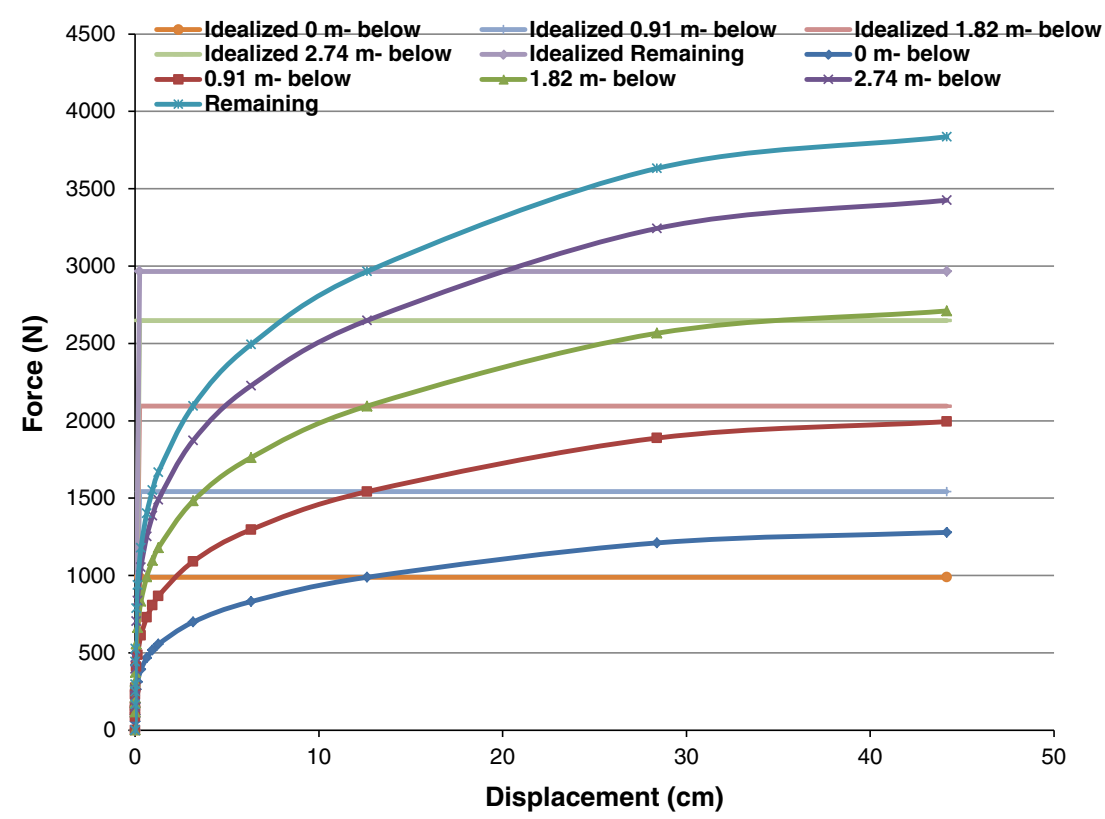

Fig. 13 Generated p-y curves at predefined locations along the depth of the pile using the FD solution by LPILE

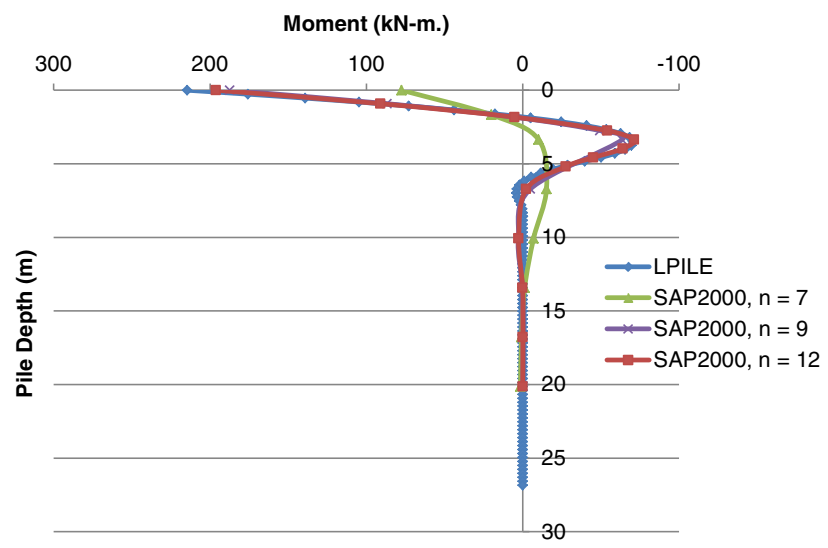

Fig. 14 Comparison between the bending moment versus pile depth obtained from the FE model SAP2000 and FD solutions by LPILE using various number of soil springs due to a lateral displacement of $2 \mathrm{~cm}$

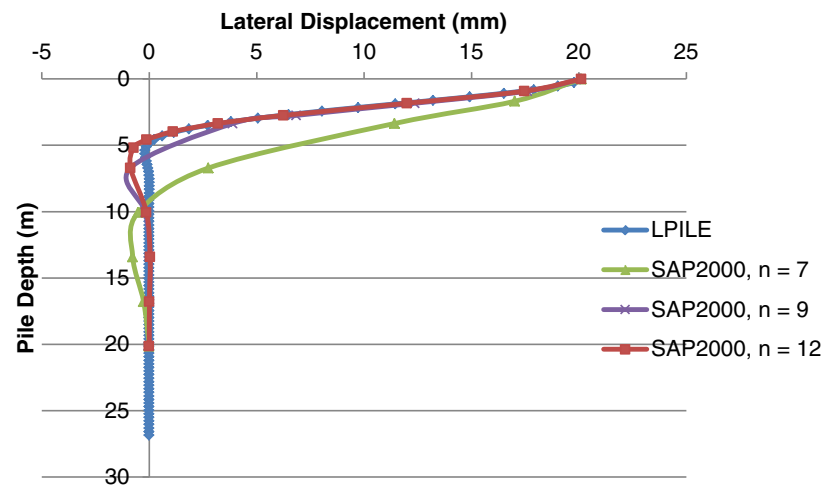

Fig. 15 Comparison between the lateral displacement versus pile depth obtained from the FE model SAP2000 and FD solutions by LPILE using various number of soil springs due to a lateral displacement of $2 \mathrm{~cm}$

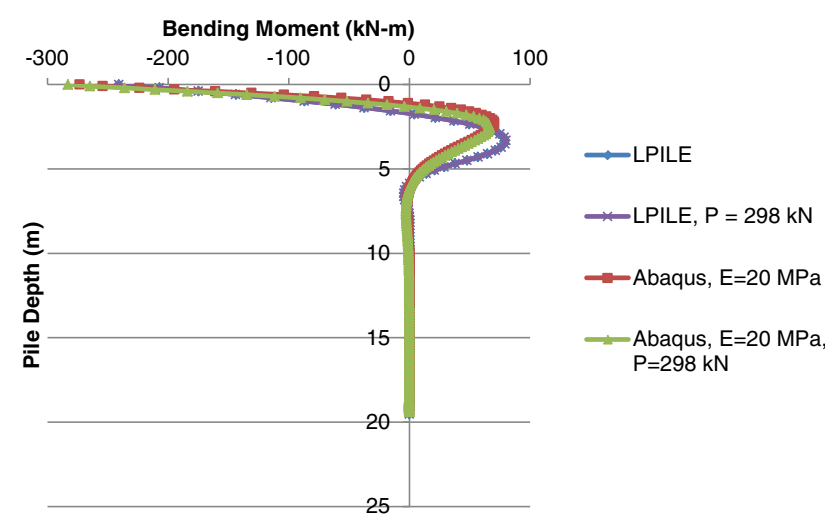

Fig. 16 Effect of axial load on the bending moment versus pile depth obtained from the FE model Abaqus/Cae and FD solutions by LPILE

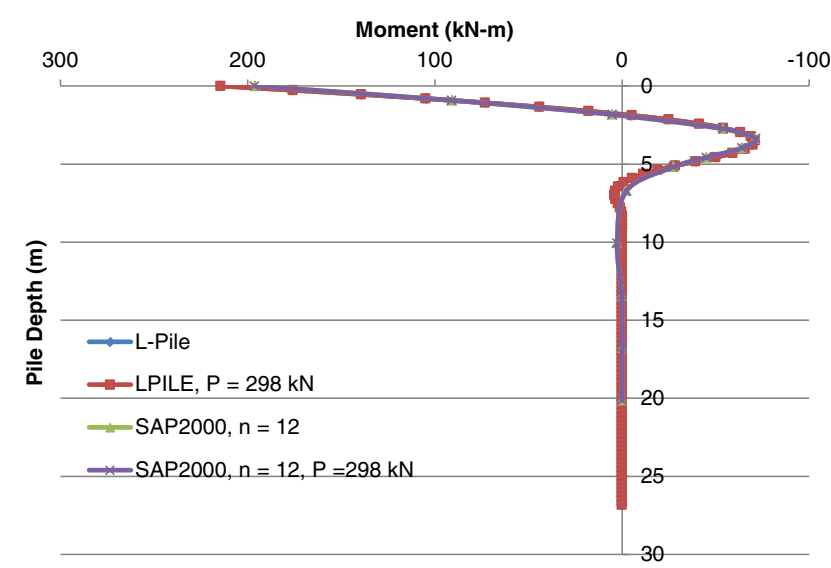

Fig. 17 Effect of axial load on the bending moment versus pile depth obtained from the FE model SAP2000 and FD solutions by LPILE 
distribution versus pile depth was similar in trend to those determined in this research.

\section{Summary and Conclusions}

The analysis of pile-soil interaction under lateral loading has always been a concern. A comparative study to analyze pile-soil interaction under lateral loading was conducted. A 2D finite difference method model was developed using LPILE, 2012. The soil was assumed to be stiff clay without free water with a unit weight of $2001.2 \mathrm{~kg} / \mathrm{m}^{3}$. The pile is oriented such that bending is about the weak axis. Two 3D finite element models were developed using the finite element software Abaqus/Cae and SAP 2000. In the 3D finite element model developed using Abaqus/Cae, both the pile and the soil were modeled using solid continuum elements (C3D8R) to account for the continuity of the soil. An elasticperfectly plastic model was adopted for the pile. A MohrCoulomb failure criterion was defined for the clay. The clay was assumed to vary from soft to hard without free water. The contact behavior between the piles and the soil was defined using tangential and normal algorithms in ABAQUS/Cae. A rigid body motion was defined at the top of the pile by tying the degrees of freedom of the elements embedded in the pile cap $(30.5 \mathrm{~cm}$ from the top of the pile) to a reference point at the centroid of the pile's cross-section. Three boundary conditions were defined into the model: (1) the bottom of the pile was fixed to model its embedment into rock below a depth of $20.12 \mathrm{~m}$ from the top of the pile, (2) the exterior surface of the soil was fixed to model its confinement at its boundaries, and (3) a displacement of $2 \mathrm{~cm}$ was applied at the top of the pile while maintaining a zero slope in what simulates a guided fixation due to the embedment of the top of the pile into the concrete pile-cap for a distance of $30.5 \mathrm{~cm}$. In the 3D finite element model developed using SAP2000, the pile was modeled using a continuum 3-D frame element while the soil was modeled using a number of nonlinear soil springs at predefined depth locations. The nonlinear soil properties were obtained using the p-y curves generated in LPILE at the predefined depth locations and modeled using the Plastic (Wen) link element available in SAP2000. A rigid body motion was defined at the top of the pile by assigning the proper degrees of freedom to the elements embedded in the concrete pile cap to maintain a zero slope in what simulates a guided fixation due to the embedment of the top of the pile into the pile-cap for a distance of $30.5 \mathrm{~cm}$. The bottom of the pile was fixed to model its embedment into rock below a depth of $20.12 \mathrm{~m}$ from the top of the pile. Also, a displacement of $2 \mathrm{~cm}$ was applied at the top of the pile.

A parametric study was conducted to examine the effect of crucial design parameters such as the variation in the magnitudes of modulus of elasticity, the amount of soil surrounding the pile, and the number of soil springs on the bending moment and lateral displacements due to an applied lateral displacement of $2 \mathrm{~cm}$ at the top of the pile. The magnitude of the modulus of elasticity was varied to reflect a variation in the stiffness of the clay from soft to hard. As the magnitude of the modulus of elasticity increases, the discrepancy between the magnitudes of the bending moment and lateral displacements induced along the depth of the pile predicted by Abaqus/Cae and those obtained from LPILE is gradually reduced to reach a minimum value when the modulus of elasticity of the soil was assumed to be 20-25 MPa which indicates medium to stiff clay.

The effect of the amount of clay surrounding the pile on the induced bending moment and lateral displacement along the depth of the pile was studied in Abaqus/Cae. The pilesoil interaction model was compared to FD solutions for a single pile embedded in clay under a displacement of $2 \mathrm{~cm}$. This is a convergence study to (1) establish the mesh density and (2) eliminate the effect of boundary condition by selecting the appropriate diameter of the soil medium around the pile. The results from FE and FD analyses showed that the discrepancy in the magnitudes of the bending moment and lateral displacements from both analyses was reduced with the increase in the amount of clay surrounding the pile. This indicates that increasing the amount of clay surrounding the piles reduces the induced bending moments and lateral displacements in the piles and thus increases its capacity to resist lateral loading. Therefore, the radius of the soil cylinder surrounding the pile was varied from 0.5 to $4 \mathrm{~m}$ to determine the most suitable soil diameter for analysis.

The effect of varying the number of soil springs on the induced bending moment and lateral displacement along the depth of the pile was examined using SAP2000. The results from SAP2000 were compared to those from FD solution by LPILE due to the effect of an induced displacement of $2 \mathrm{~cm}$ at the top of the pile. The number of nonlinear soil springs was varied between 7, 9, and 12 springs. Using a larger number of nonlinear soil springs showed a better agreement between bending moment and lateral displacement magnitudes obtained using SAP2000 and LPILE.

The results obtained from the FE models and FD solutions show that SAP2000 was capable of predicting the induced bending moments and lateral displacements along the depth of the pile more closely than Abaqus/Cae. The reason for that can be attributed to the nature of the soil definition in the finite element models. In SAP2000, the soil is defined as isolated springs, which is similar to the soil definition in LPILE, and the soil stiffness obtained from LPILE was used into SAP2000 which resulted in obtaining almost a perfect match for the bending moment and the lateral displacement curves. However, the soil definition in Abaqus/Cae is based on a soil continuum model which resulted in a discrepancy between the results obtained by LPILE and those calculated by Abaqus/Cae. Overall, the results of Abaqus/Cae are considered to be in a good agreement with those of LPILE.

Also, the effect of applying an axial load of $298 \mathrm{kN}$ to the pile on the produced bending moment and lateral displacement along the depth of the pile due to the applied displacement of $2 \mathrm{~cm}$ at the top of the pile is minimal and can be neglected. 


\section{Recommendations}

1. An agreement between the results of LPILE, SAP2000, and Abaqus/Cae was achieved. It is recommended that a design engineer may use LPILE to predict pile-soil interaction.

2. If SAP2000 is used, it is recommended that a design engineer may use the largest number possible of springs, similar to what is used in this study.

3. It is recommended to investigate and compare the pilesoil interaction in a single pile against that of pile-bent subjected to axial and lateral loads. It will be important to study the effect of a wide range of important design parameters. This comparison will inform design engineers of the difference in pile-soil interaction between a single pile and a group of piles.

4. It is recommended to design and conduct an experimental study to test a single pile in soft and stiff soil under the effect of axial and lateral loads.

\section{Open Access}

This article is distributed under the terms of the Creative Commons Attribution License which permits any use, distribution, and reproduction in any medium, provided the original author(s) and the source are credited.

\section{References}

Abdel-Mohti, A., \& Pekcan, G. (2013a). Effect of skew on the seismic vulnerability of RC box girder highway bridges. International Journal of Stability and Sturctural Dynamics, 13(6).

Abdel-Mohti, A., \& Pekcan, G. (2013b). Assessment of seismic performance of skew reinforced concrete box girder bridges. International Journal of Advanced Structural Engineering, 5(1).

Arsoy, S., Barker, R. M., \& Duncan, J. M. (1999). “The behavior of integral abutment bridges.” VTRC 00-CR3. Virginia Transportation Research Council, Charlottesville, VA.

Bijnagte, J. L., Van Den Berg P., Zorn, N. F., \& Dieterman, H. A. (1991). "Laterally loaded single piles in soft soil-theory and reality.” HERON, 36 (1), Jointly edited by STEVIN Laboratory of the Faculty of Civil Engineering, Delft University of Technology, Delft, and TNO Building and Construction Research, Rijswijk, Netherlands, pp 78.

Broms, B. B. (1964). Lateral resistance of piles in cohesionless soils. Journal of Soil Mechanics and Foundation Division ASCE, 90(3), 136-156.

Brown Dan, A., \& Shie, C.-F. (1990). Three dimensional finite element model of laterally loaded piles. Computers and Geotechnics, 10(1), 59-79.

Brown Dan, A., \& Shie, C.-F. (1991). Some numerical experiments with a three dimensional finite element model of a laterally loaded pile. Computers and Geotechnics, 12(2), 149-162.

Desai, C. S., \& Appel, G. C. (1976). 3-D analysis of laterally loaded structures. Second International Journal Conference on Numerical Methods in Geomechanics, Blacksburg, VA, ASCE, 1, 405-418.

Desai, C. S., \& Kuppusamy, T. (1980). Application of a numerical procedure for laterally loaded structures. Numerical Methods in Offshore Piling ICE, 1980, 93-99.

Dunnavant, T. W., \& O’Neill, M. W. (1989). Experimental p-y model for submerged, stiff clay. Journal of Geotechnical Engineering, 115(1), 95-114.

Ellis, E. A., \& Springman, S. M. (2001). Modeling of soilstructure interaction for a piled bridge abutment in plain strain FEM analyses. Computers and Geotechnics, 28(2), 79-98.

Faraji, S., Ting, J. M., Crovo, D. S., \& Ernst, H. (2001). Nonlinear analysis of integral bridges: finite-element model. Journal of Geotechnical and Geoenvironmental Engineering, 127(5), 454-461.

Faruque, M. O., \& Desai, C. S. (1982). "3-D material and geometric non-linear analysis of piles.” Proceedings of the Second International Conference on Numerical Methods for Offshore Piling, Austin, TX.

Gabr, M. A., Lunne, T., \& Powell, J. J. (1994). P-Y analysis of laterally loaded piles in clay using DMT. Journal of Geotechnical Engineering, 120(5), 816-837.

Georgiadis, M., \& Butterfield, R. (1982). Laterally loaded pile behavior. Journal of Geotechnical Engineering Division ASCE, 108(GT1), 155-165.

Greimann, L. F., Yang, P. S., \& Wolde-Tinsae, A. M. (1986). Nonlinear analysis of integral abutment bridges. Journal of Structural Engineering, 112(10), 2263-2280.

Hetenyi, M. (1946). Beams on elastic foundation. Ann Arbor: The University of Michigan Press.

Khodair, Y., \& Hassiotis, S. (2013). Rigidity of abutments in integral abutment bridges. Journal of Structure and Infrastructure Engineering, Maintenance, Management, LifeCycle Design and Performance, 9(2), 151-160.

Kim, Y., \& Jeong, S. (2011). Analysis of soil resistance on laterally loaded piles based on 3D soil-pile interaction. Computers and Getechnics, 38, 248-257.

Kooijman, A. P. (1989). Comparison of an elasto-plastic quasi three-dimensional model for laterally loaded piles with field tests. In S. Pietruszczak \& G. N. Pande (Eds.), Numerical models in geomechanics-NUMOG III (pp. 675-682). New York, NY: Elsvier Applied Science Publishers.

Kumar, B. S. (1992). "Three-dimensional non-linear finite element analysis of laterally loaded piles in clay." Ph.D. Thesis Dissertation, University of Illinois at UrbanaChampaign, IL.

McCelland, B., \& Focht, J. A. (1958). Soil modulus for laterally loaded piles. Transactions ASCE, 123, 1049.

O’Neill, M. W., \& Gazioglu, S, M. (1984). "An evaluation of p-y relationships in clays.” A Report to the American Petroleum Institute, PRAC82-41-2, The University of Houston-University Park, Houston, TX 
Rajashree, S. S., \& Sitharam, T. G. (2001). Nonlinear finiteelement modeling of batter piles under lateral load. Journal of Geotechnical and Geoenvironmental Engineering, 127(7), 604-612.

Reese, L. C., \& Matlock, H. (1956). "Non-dimensional solutions for laterally loaded piles with soil modulus assumed proportional to depth.” Proceedings of the 8th Texas Conference on Soil Mechanics and Foundation Engineering, Sp. Pub. 29, Bureau of Engineering Research, University of Texas, Austin, TX.

Robertson, P. K., Campanella, R. G., Brown, P. T., Grof, I., \& Hughes, J. M. O. (1985). "Design of axially and laterally loaded piles using in-situ tests: a case history.” Canadian Geotechnical Conference, pp. 51-60.

Ruesta, P. F., \& Townsend, F. C. (1997). Evaluation of laterally loaded pile group at Roosevelt bridge. Journal of Geotechnical and Geoenvironmental Engineering, 123(12), 1153-1161.

Thompson, G. R. (1977). “Application of finite element method to the development of $\mathrm{p}$-y curves for saturated clays, M.S. Thesis, University of Texas, Austin, TX, pp. 190. 